\title{
Stable geographic forwarding with link lifetime prediction in mobile adhoc networks for battlefield environment
}

\author{
Priyanka Jaiswal and Adwitiya Sinha* (1)
}

\author{
*Correspondence: \\ mailtoadwitiya@gmail.com \\ Department of Computer \\ Science \& Engineering, \\ Jaypee Institute \\ of Information Technology, \\ Noida, India
}

\begin{abstract}
Mobile adhoc network (MANET) is one of the most relevant areas of research in wireless communication that has gained prevalence due to its diversity over large-scale highly mobile networks to small-scale networks having low mobility and power constraints. Adhoc networks are inherently envisioned to be highly vulnerable to dynamic changes because of mobile sensor nodes. Node mobility often results in breakage of communication links, thereby introducing additional overhead for establishing new routes and transmitting table updates, further causing rapid exhaustion of energy reserve. Especially in military applications, preventing communication disruptions is an important security concern for defence applications engaged in safeguarding national boundaries. This necessitates the need for efficient routing strategy for battlefield environments susceptible to frequent link failures due to random mobility of groups/ individuals. In this regard, we have proposed an efficient stable geographic forwarding with link-lifetime prediction (SGFL) that utilizes the broadcast nature of wireless channel and multicasts with node mobility. During the next hop selection process, a node preferably selects the neighbours which are at the least distance from the destination with low mobility. Unlike position based opportunistic routing, our scheme allows selection of backup node that lies within the transmission range of selected neighbours. Link lifetime prediction with backup nodes enhances efficiency and reliability of routing in highly mobile and congested adhoc networks. Simulation results show that our proposed SGFL achieves better performance than existing counterparts in terms of packet delivery ratio, packet loss and end-to-end delay under high node density as well as increased traffic flow.
\end{abstract}

Keywords: Mobile adhoc networks, Geographic forwarding, Link stability, Backup routing, Link prediction, Network performance, Energy consumption

\section{Background}

Adhoc network consists of mobile sensor nodes distributed across a geographical region, performing wireless communication to interact with each other. In case the host sensor is not in the direct range of its destined location, it preferably forms multi-hop network via intermediate nodes in absence of any central coordination or infrastructure. These networks are mostly predominant in applications where rapid deployment is required,

(c) 2016 The Author(s). This article is distributed under the terms of the Creative Commons Attribution 4.0 International License (http://creativecommons.org/licenses/by/4.0/), which permits unrestricted use, distribution, and reproduction in any medium, provided you give appropriate credit to the original author(s) and the source, provide a link to the Creative Commons license, and indicate if changes were made. 
for instance, battlefield surveillance, disaster relief services, medical consultation in remote areas, etc.

On the basis of the type of information used in packet routing, Mobile adhoc network (MANET) routing protocols are categorized as topology-based and position-based protocols $[1,2]$. The topology-based protocols utilize link information from the network to forward packet in a proactive or reactive manner. In proactive routing, all paths are computed before packet forwarding initiates, hence increasing network overhead. Reactive routing allows computation of the single path required for communication. This reduces route computation overhead but increases the delay incurred in performing route discovery. Second category of protocols belonging to position-based routing relies on the knowledge of geographical position of the nodes instead of network addresses [3]. In this scheme, each node receives its position information through location service techniques (or global positioning devices) for forwarding packets efficiently towards its intended destination. Therefore, geographic routing alleviates the need for maintaining route information. This further reduces message exchanges resulting from table updates, thereby automatically mitigating network overhead as compared to topology-based routing. Greedy forwarding is another basic scheme that is used in geographic routing to relay packets [4]. This strategy allows source nodes to select next-hop from neighbour list that lies in the progress area towards destination node. In greedy routing, communication link is available only when node lies in the progress area.

\section{Problem definition and motivation}

Due to high node mobility the neighbour list of a node might change more often, thereby raising the need for predicting link lifetime for improving reliability in communication. The cases when network size is large or traffic rate is very high, often leads to frequent congestion in the network. Moreover, when nodes attempt to update their location information, then there is a higher probability for loss of beacon messages due to congested network. In such situation, the node fails to receive acknowledgement for its beacon messages and assumes that its current neighbour list has got invalidated. This initiates search for new neighbours to forward data which ultimately causes tremendous increase in delays involved in delivering network services.

These circumstances are mostly prevalent in battlefield operations, where each person uses wireless device having limited battery power and each missile vehicle are wireless enabled with higher power reserve. Therefore, energy-aware routing becomes an issue of prime importance for the armed individuals relying on restricted powered wireless devices. Wastage of radio resources and battery lifetime leads to degradation of network performance. This further encourages design of efficient routing protocols that can provide reliable and efficient data delivery against node mobility and dynamic topology.

\section{Research overview}

There are several routing schemes based on greedy forwarding, can be employed in this regard. However, greedy forwarding applies only distance metric to select next forwarder which is not enough for high mobility and congested network scenario. For this purpose, an additional metric along with distance parameter is required for improving 
efficiency of routing by maximizing packet delivery with reduced delay and energy consumption for both the scenarios.

In our research, we have proposed link stability metric with distance metric to forward packet from between a pair of communicating nodes. This helps the source node to identify links that are more stable than others during next forwarder selection. Communication over stable links reduces packet loss because of link failure. The proposed scheme employs backup node along with the next forwarder node that prevents interruption in communication even if the link fails. Our method considers the position, speed and distance of sensor node (i.e. the armed person carrying the sensor).

The rest of the paper is organized as follows: "Background" section presents an overview of the existing link lifetime estimation methods and stability based routing protocols. "Related work" section highlights the network model assumptions for battlefield environment. Our proposed routing protocol with link stability prediction is proposed in "Proposed backup routing protocol based on link lifetime prediction" section. In "Performance evaluation" section, the performance of proposed SGFL is validated through simulation results. Finally, "Conclusion" section concludes with our research with future directions.

\section{Related work}

In MANET, researchers have developed numerous methods for estimating link lifetime, some of them are lifetime based or mobility based mechanism to calculate the stability of link between pair of node. Link stability refers to the duration for which connectivity between a pair of nodes exists. Several mobility models for adhoc networks have been described, for instance, random walk mobility, random waypoint mobility and random group mobility models [5]. In battlefield environment, individuals (sensors) move independently or in group. Such military applications require continued connectivity with link stability [6-12]. In Greedy based forwarding schemes, link is considered to be stable when pair of nodes lies in the progress area of source node. A neighbouring node lies in the progress area if distance from source to destination is greater than distance between neighbour nodes to destination node. Power boosting geographic routing (PBGR) [13] is greedy forwarding technique that performs link lifetime estimation to select next forwarder node. When a dead node problem occurs then PBGR increase its node transmission range to find neighbour node. It involves complex relation between power consumption and node interference range. Another scheme illustrated in [14], greedy perimeter stateless routing with lifetime (GPSR-L) is a combination of greedy routing and face routing, which further analyses the lifetime between source and neighbour node, and accordingly set the lifetime timer for each of its neighbour. The neighbour node having least distance from destination and largest timer value is selected as next forwarder node to forward packet. The results show that GPSR-L improves network performance for both high mobility and congested scenario. However in case of void area or dead node, absence of neighbours in the progress area of source node towards destination makes it necessary to switch from greedy mode to perimeter mode. As a result, this increases the hop count and introduces additional overhead and wastage of sensor energy. 
In [15] authors have proposed stability aware greedy (SAG) routing that calculates link lifetime metric using node position, speed and direction for each node and finally selects highest link lifetime stable node as next forwarder node. When sending node detects link failure using beacon message then source node searches for new node to designate it as the next forwarder. Another scheme presented in [16], greedy forwarding with more reliability (MFR) that two other metrics besides distance metric, first is velocity similarity metric which select neighbour which has similar speed with sending node and second is power similarity metric that selects neighbour based on power similarity with sending node. However, in battlefield MFR is not suitable because it requires time sensitive application. The authors of [17] proposed link stability and path stability based routing to select path with minimum hop count. The distance between two adjacent nodes is computed using log distance path loss model. When the distance between two neighbour nodes is more than stable zone of the communication range then re-routing is initiated before the route fails.

In order to improve the reliability of a communication system in military application, the most effective concept is to increase the degree of redundancy. There are several existing robust routing protocol and can be categorized as end-to-end redundancy (e.g. multipath routing) and hop-by-hop redundancy (e.g. opportunistic forwarding), which takes the advantage of wireless channel and multicasting to forward packets. Position based opportunistic routing (POR) [18] is an instance of opportunistic forwarding. POR follows greedy forwarding scheme to transmit packets and make the forwarding list that includes next forwarder node along with several backup nodes. When next forwarder fails to transmit the packet then backup node replaces to continue transmission. POR performs better in highly mobile scenario where network size is small or traffic rate is slow. Link lifetime based backup routing (LBR) [19] uses multipath scheme with adhoc on demand distance vector (AODV) routing to improve route stability. LBR determines the shortest path between source and destination through flooding and creates backup path locally for each link with primary path. Every link in the primary path is selected with respect to the link lifetime estimation. When primary path fails then local backup path takes place to continue the communication. In yet another research conducted in [20], authors developed link lifetime-based segment-by-segment routing protocol (LLSSR). The strategy allows the selection of a stable route segment-wise to reduce the overhead of routing protocol with each node maintaining a routing table for its k-hop region.

In order to improve link stability in situation where link quality is poor due to congestion and highly mobile environment (e.g., military scenario), we present an efficient routing protocol, stable geographic forwarding with link-lifetime prediction (SGFL). The major contributions are enlisted as following:

1. The research aims to build a reliable scheme to route communication for critical applications, including defence and battlefield services that demands reliability in both, connectivity and continuity. 
2. The scheme involves building protocol to establish geographic forwarding with link prediction and stability.

3. It uses link stability metric with respect to least distance and low mobility of nodes lying in the progress area of source node, for selecting next forwarder.

4. In addition, a stable backup node is designated along with the next forwarder, so that in case of link breakage, the backup node can forward transmission without further delays.

5. The performance of our proposed scheme nomenclatured as Stable Geographic Forwarding with Link-lifetime prediction (SGFL) was established on the basis of network performance parameters, involving packet delivery ratio, end-to-end delay, routing overhead and energy consumption.

6. Comparative study of proposed SGFL is performed with existing counterpart mechanisms, including heading-direction angles routing protocol (HARP) [17], POR [18], and LBR [19] respectively.

\section{Proposed backup routing protocol based on link lifetime prediction}

Link and route stability is a major issue in mobile networks, specifically in military applications where all the nodes possess different speed and has highly mobile as well as congested environment $[21,22]$. In this regard, we propose SGFL routing algorithm to transmit packets over stable links. Our SGFL routing protocol is based on greedy forwarding scheme, where the source node only forward packet to its neighbour node that lies closest towards the destination in progress area. This procedure is carried out for each selected forwarding node until the destination is reached. Source and destination nodes are denoted by $S$ and $D$ respectively as shown in Fig. 1 along with the forwarding area highlighted by dotted lines.

We propose a next hop selection metric that is based on the stability of links among mobile nodes. Stability of link is denoted as a function of the least distance from destination and lower mobility than neighbour nodes with respect to the source node. The

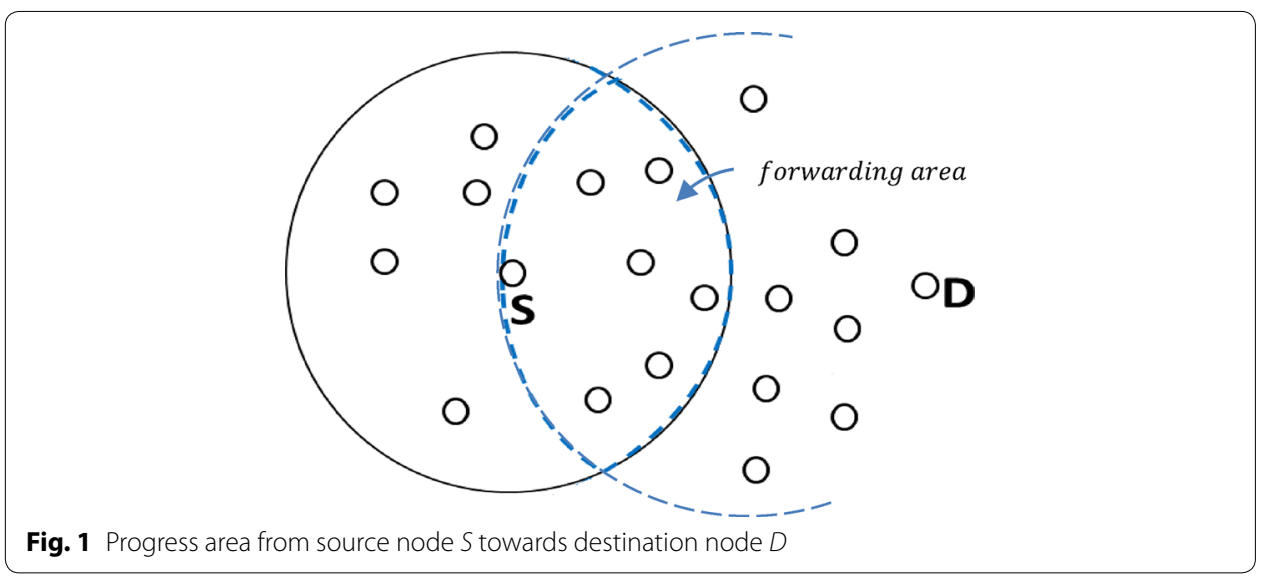


selected next-forwarder will be responsible for choosing its backup node and forwarding the packet to its next-forwarder. Only the nodes located in the progress area and half of communication range of next- forwarder would get the chance to be elected as backup node. A neighbour node lies in the progress area if and only if it satisfies the two conditions: (i) firstly, distance between each node from source should not exceed the communication range $R$ of source node and (ii) secondly, distance between neighbour nodes to destination node should not exceed from distance between source nodes to destination node. Three algorithms are proposed, (1) Packet Forwarding Strategy, (2) Next-hop forwarder selection and (3) backup node selection approach. Source node initially executes algorithm 1 if there is no neighbour between source and destination node to forward packet, otherwise directly executes algorithm 2 and 3.

According to algorithm 2 source node gets its neighbour nodes list $N b r(S)$ lying within its transmission radius $R$. This is followed by selection of maximum two neighbours with least distance, $N_{l}$ from destination node $D$ using distance comparison function $(\Delta(x, y))$, as shown in the following Eqs. (1-3):

$$
\begin{aligned}
& \operatorname{Nbr}(S)=\left\{n_{i}, n_{i+1}, \ldots n_{n} \mid R\left(n_{i}\right)<R(S)\right\} \\
& N_{l}=\min _{\Delta}\{\operatorname{Nbr}(S)\} \\
& n_{h}=\min _{\delta}\left\{N_{l}\right\}
\end{aligned}
$$

After the source node finds the neighbour node from its progress area, it then applies speed comparison function $\delta(x)$. An example shown in Fig. 2 where node $n_{2}$ and node $n_{3}$ has least distance from destination and lies in progress area of nodes $S$. For instance, if node $n_{2}$ bears a speed of $9 \mathrm{~m} / \mathrm{s}$ and node $n_{3}$ moves with speed of $1 \mathrm{~m} / \mathrm{s}$ that is slower than node $n_{2}$, then, $n_{3}$ has lesser probability to move out of communication range of sending node $S$. Therefore, node $n_{3}$ is chosen as the next highly stable forwarder. This is followed by execution of algorithm 3 for discovering backup node. Since there is assumption that

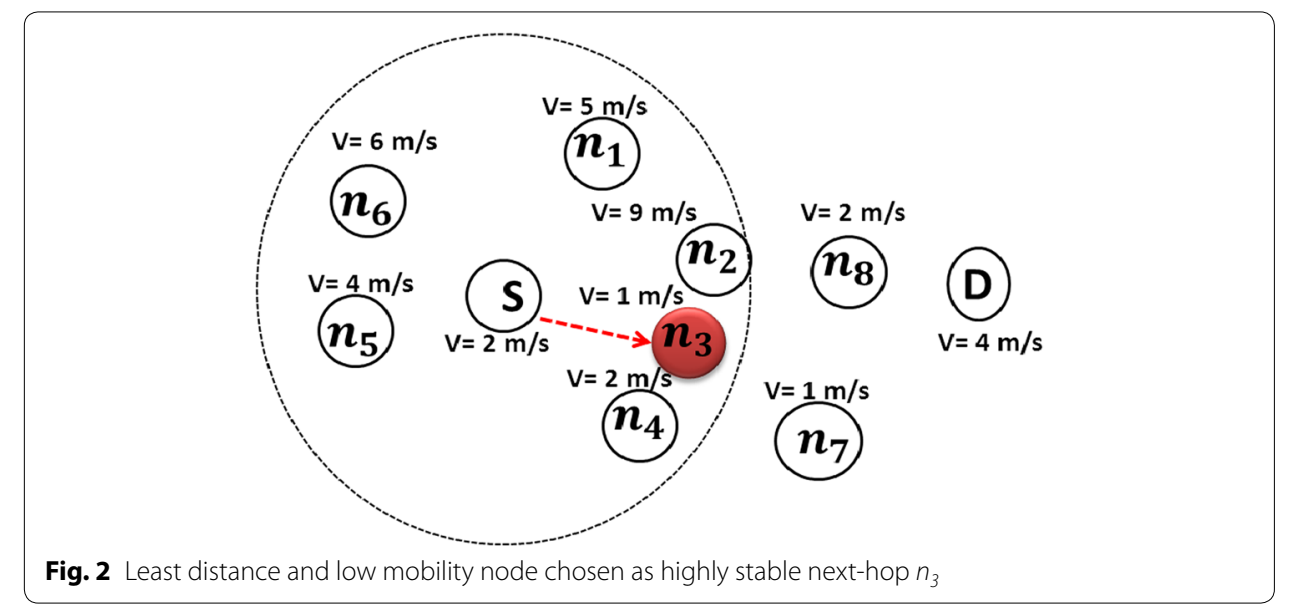


nodes are highly mobile and congested, therefore Next forwarder node finds its backup node based on link stability metric using distance comparison function $(\Delta(x, y))$ and speed comparison function $\delta(x)$, as shown in Eqs. (4-5):

$$
\begin{aligned}
& N b r\left(n_{h}\right)=\left\{n_{i}, n_{i+1}, \ldots n_{n} \mid R\left(n_{i}\right)<R\left(n_{h}\right) / 2\right\} \\
& N_{l b}=\min _{\Delta}\left\{\operatorname{Nbr}\left(n_{h}\right)\right\} \quad \text { where } N_{l} \leq 2 \\
& n_{b}=\min _{\delta}\left\{N_{l b}\right\}
\end{aligned}
$$

Source node sends packet to next forwarder node $n_{h}$ as well as backup node $n_{b}$ simultaneously as depicted by Eq. (7) for next-hop transmission:

$$
C_{S+i}=\left\{n_{h} \cup n_{b}\right\}
$$

An example is illustrated in Fig. 3, where node $n_{3}$ is next forwarder and it finds its backup node within its half communication radius. Node $n_{4}$ is chosen next highly stable backup node. When the sending node detects through beacon message that link is failed, then backup node is adopted to continue packet transmission without incurring further delay.

Through this mechanism, SGFL maintains link and route lifetime efficiently in battlefield environment to support group mobility pattern (Fig. 4). Suppose a message is send
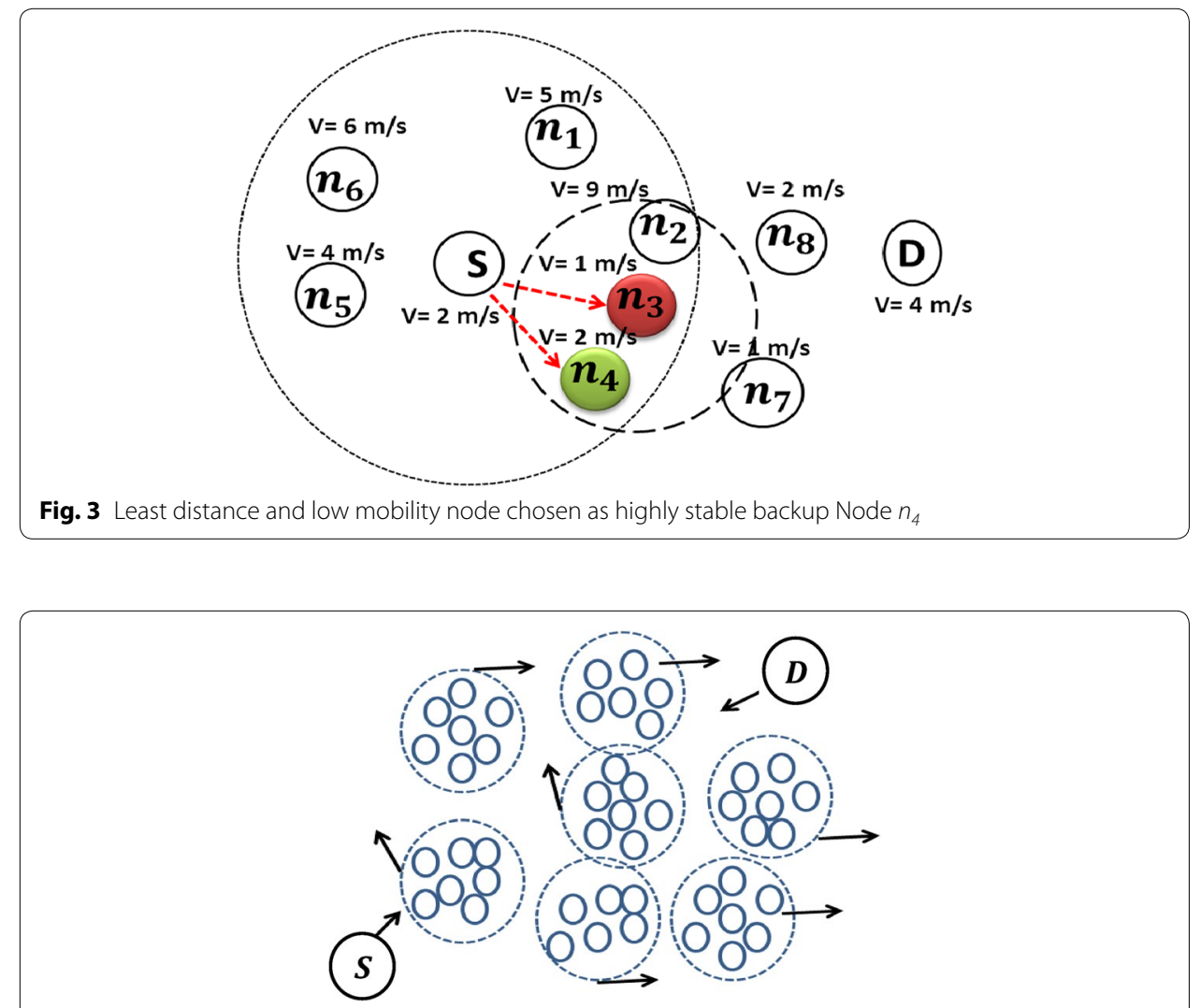

Fig. 4 Scenario 1 using random group mobility model 
by a base camp or leader in battlefield to mobile nodes which can be a person or can be vehicles and person has been killed or tankers have been destroyed. In that case there is need of backup nodes to continue transmission. Therefore, our proposed SGFL protocol copes with the resource constraints in a mobile adhoc battlefield network and improves stability for node communications with low energy consumption. The notations used in algorithms are summarized in Table 1.

\section{Performance evaluation}

In this section, we evaluated the performance of our proposed method SGFL using NS2.34 simulator [23-25]. Each node is assumed to find its location using positioning system, like GPS or suitable location service. The performance evaluation metrics include, packet delivery ratio, routing overhead, end-to-end delay, and energy

Table 1 Algorithmic notations

\begin{tabular}{ll}
\hline Notation & Meaning \\
\hline$S$ & Source or sender node \\
$D$ & Destination or receiving node \\
ListN & List of neighbours \\
ListS & List of sorted neighbour based on distance form destination \\
ListB & List of all backup nodes of next forwarder which is initially zero \\
$\Delta\left(N_{i}, N_{j}\right)$ & Function that return distance between $N_{i}$ and $N_{j}$ \\
$\delta\left(N_{j}\right)$ & Function that return speed of node $N_{i}$ \\
$n_{h}$ & Next-hop forwarder node obtained from NextForwarder() \\
$n_{b}$ & Backup node used when next hop fails to transmit packet, acquired as outcome from BackupNode() \\
\hline
\end{tabular}

\section{Algorithm 1: Packet Forwarding Strategy}

Input: $\operatorname{ListN}$, sender $(S)$, destination $(D), \operatorname{ListB}=0$

\section{Procedure:}

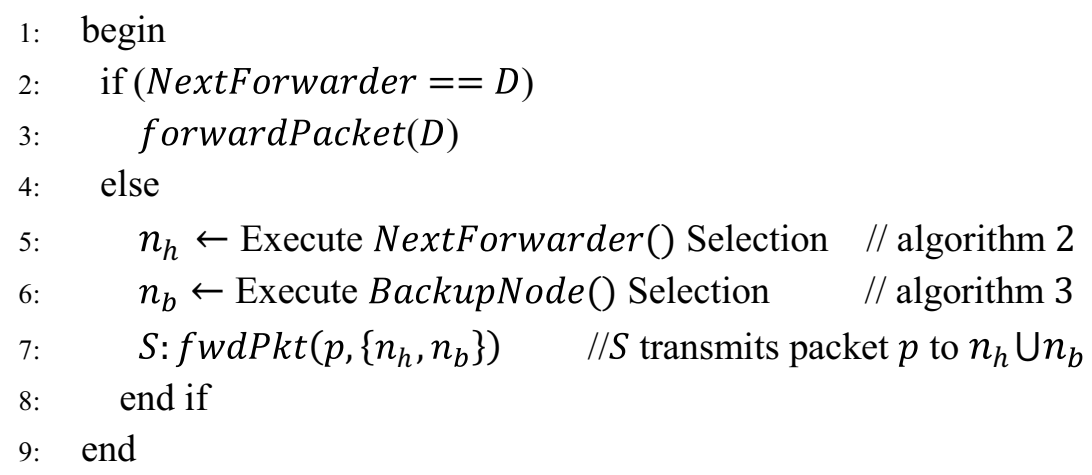




\section{Algorithm 2: Next-hop Forwarder Selection, NextForwarder ()}

Input: ListN, sender $(S)$, destination $(D)$

\section{Procedure:}

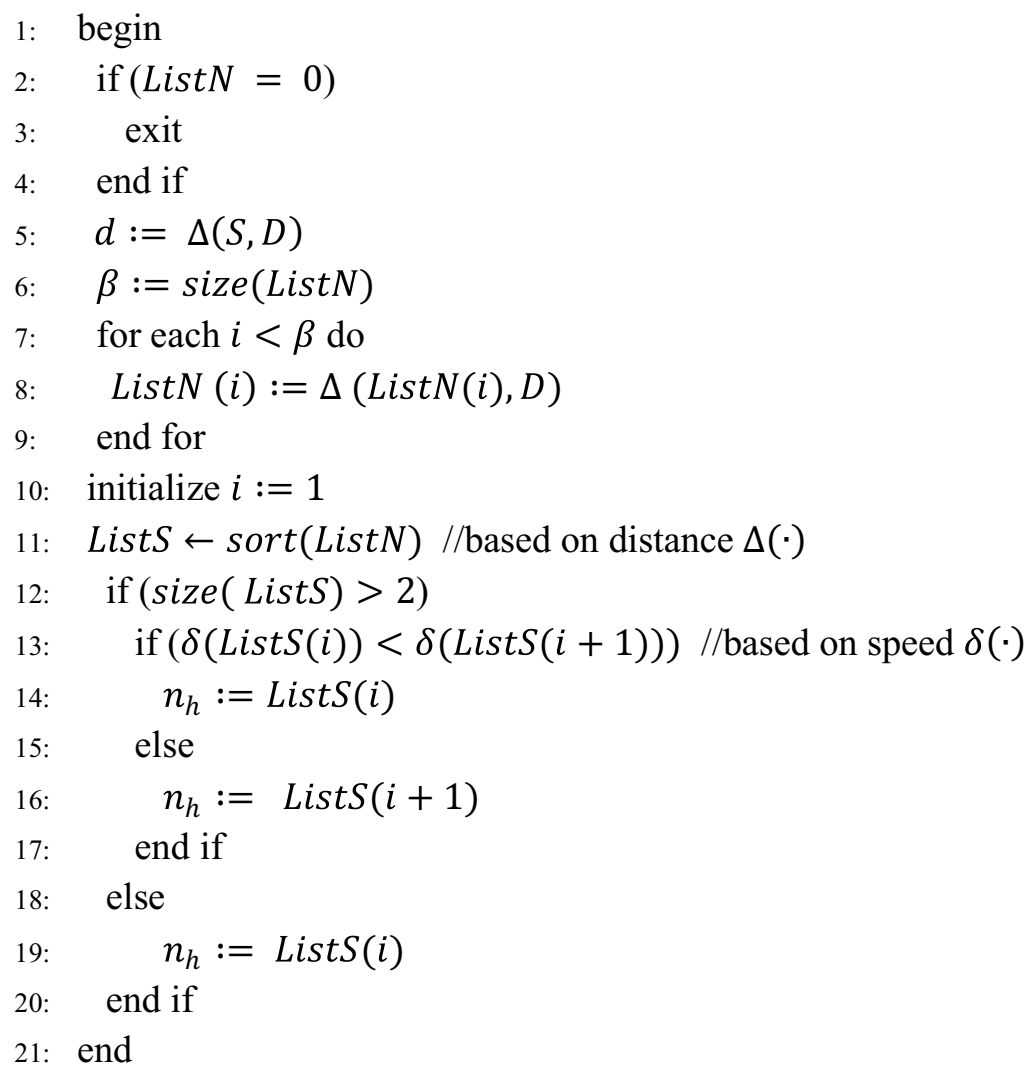

Output: ListS, $\beta, n_{h}$

consumption w.r.t. network traffic rate and node mobility. For experimentation, two different network scenarios are taken and simulation parameters are initialized as in Table 2. In the first scenario, source and destination node are assumed to move towards each other and intermediate nodes spread between source and destination region, move at random speed with random group mobility model as shown in Fig. 4. Average of 8 executions is taken w.r.t. network traffic rate varying between 10 to 100 packet/s. All nodes have their maximum speed from $50 \mathrm{~m} / \mathrm{s}$ at the time of simulation with $2 \mathrm{~s}$ pause time.

In the second scenario, source and destination as well as intermediate nodes are moving at random speed with random way point mobility model. Intermediate nodes are deployed between source and destination region as shown in Fig. 5. An average 8 
Algorithm 3: Backup Node Selection, BackupNode()

Input: ListS, $n_{h}, R$

\section{Procedure:}

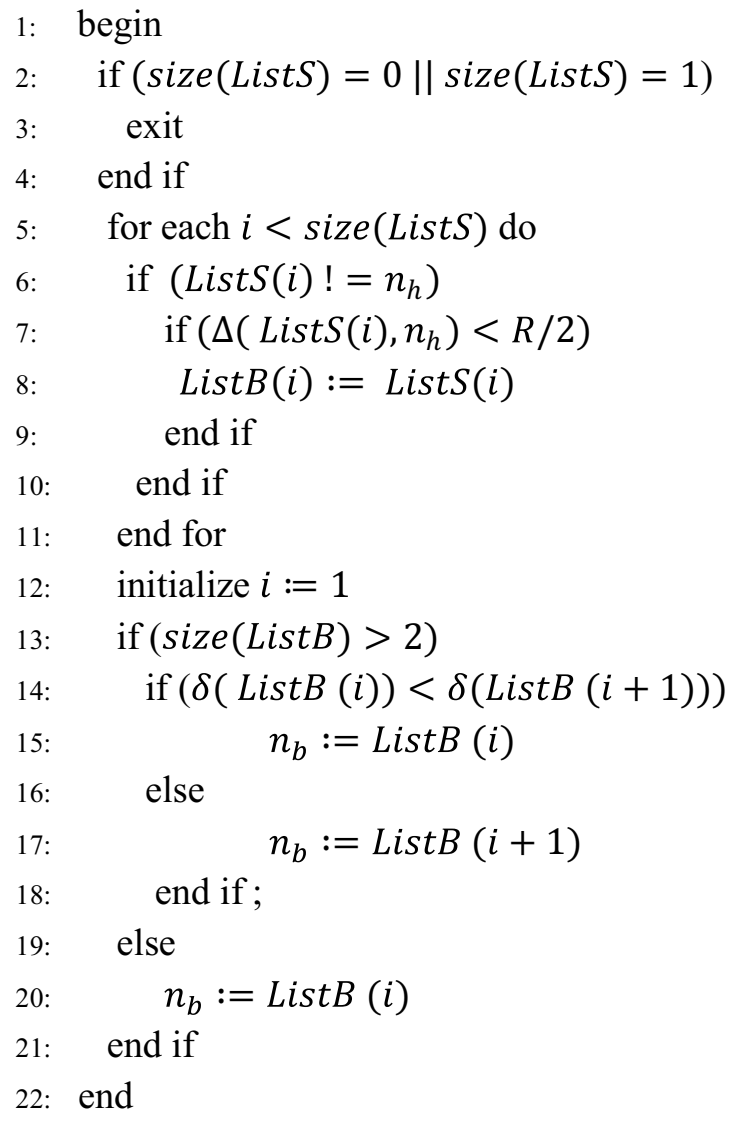

\section{Output: $\operatorname{List} B, n_{b}$}

executions taken into considered w.r.t. network traffic rate varies from 10 to $100 \mathrm{p} / \mathrm{s}$. All nodes have their maximum speed from $50 \mathrm{~m} / \mathrm{s}$ at the time of simulation with $1 \mathrm{~s}$ pause time.

\section{Impact of network traffic on individual and group mobility scenarios}

This section includes the impact of network traffic on first scenario having group mobility (Fig. 6) and second scenario with random individual node mobility (Fig. 7). Figures 6a and $7 \mathrm{a}$ show the packet delivery ratio achieved by our proposed SGFL and existing POR [18], respectively. It is clearly apparent from the results that SGFL achieves higher delivery ratio which eventually decreases as the network traffic rate increases. Packet 
Table 2 Simulation parameters

\begin{tabular}{ll}
\hline Parameter & Initialization \\
\hline MAC protocol & IEEE 802.11 \\
Transmission range & $200 \mathrm{~m}$ \\
Mobility model & Random group mobility model (RGM), random way point model (RWP) \\
Traffic rate & $10-100$ packet/s \\
Packet size & 1024 byte \\
Number of nodes & 80 \\
Simulation time & $50 \mathrm{~s}$ \\
Antenna type & Omnidirectional \\
Transport agent & UDP \\
Speed of node & $50 \mathrm{~m} / \mathrm{s}$ \\
Pause time & $1,2 \mathrm{~s}$ \\
Energy model & Radio energy model \\
Initial energy & $100 \mathrm{~J}$ \\
Transmission power & $1.0 \mathrm{~W}$ \\
Reception power & $1.0 \mathrm{~W}$ \\
Sleep time & $.001 \mathrm{~s}$ \\
Transmission time & $.005 \mathrm{~s}$ \\
\hline
\end{tabular}

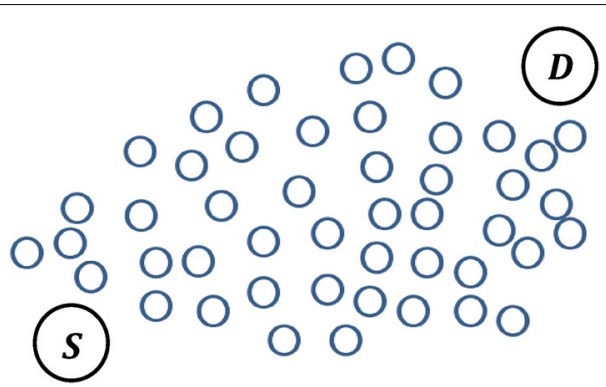

Fig. 5 Scenario 2 using random way point mobility model

overhead increases as CBR packet frequency are increased, thus the network becomes more congested and data packets are dropped, which leads to a decreased packet delivery ratio. However, SGFL has a comparatively higher packet delivery ratio than POR and shows a slower decreasing tendency for both the scenarios. This is because of the fact that link lifetime is proposed in SGFL which has highly stable nodes in SGFL, which reduces the number of dropped packet as compared to POR. Figures $6 \mathrm{~b}$ and $7 \mathrm{~b}$ show the variation of end-to-end delay with respect to CBR packet frequency. As the number of CBR frequency increases, transmission delay is increased. That is because network overhead is increased as the number of CBR frequency increase, and the network becomes more congested, which leads to increase in end-to-end delay. However end-to-end delay for proposed SGFL exhibits a slower trend because of the usage of improved link stability parameter.

Figures $6 \mathrm{c}$ and $7 \mathrm{c}$ show the variation of packet rate with respect to routing overhead in terms of total number of sent and forwarded packets in routing process with the increase of CBR packet frequency in scenario 1 and 2. As the number of CBR packet 


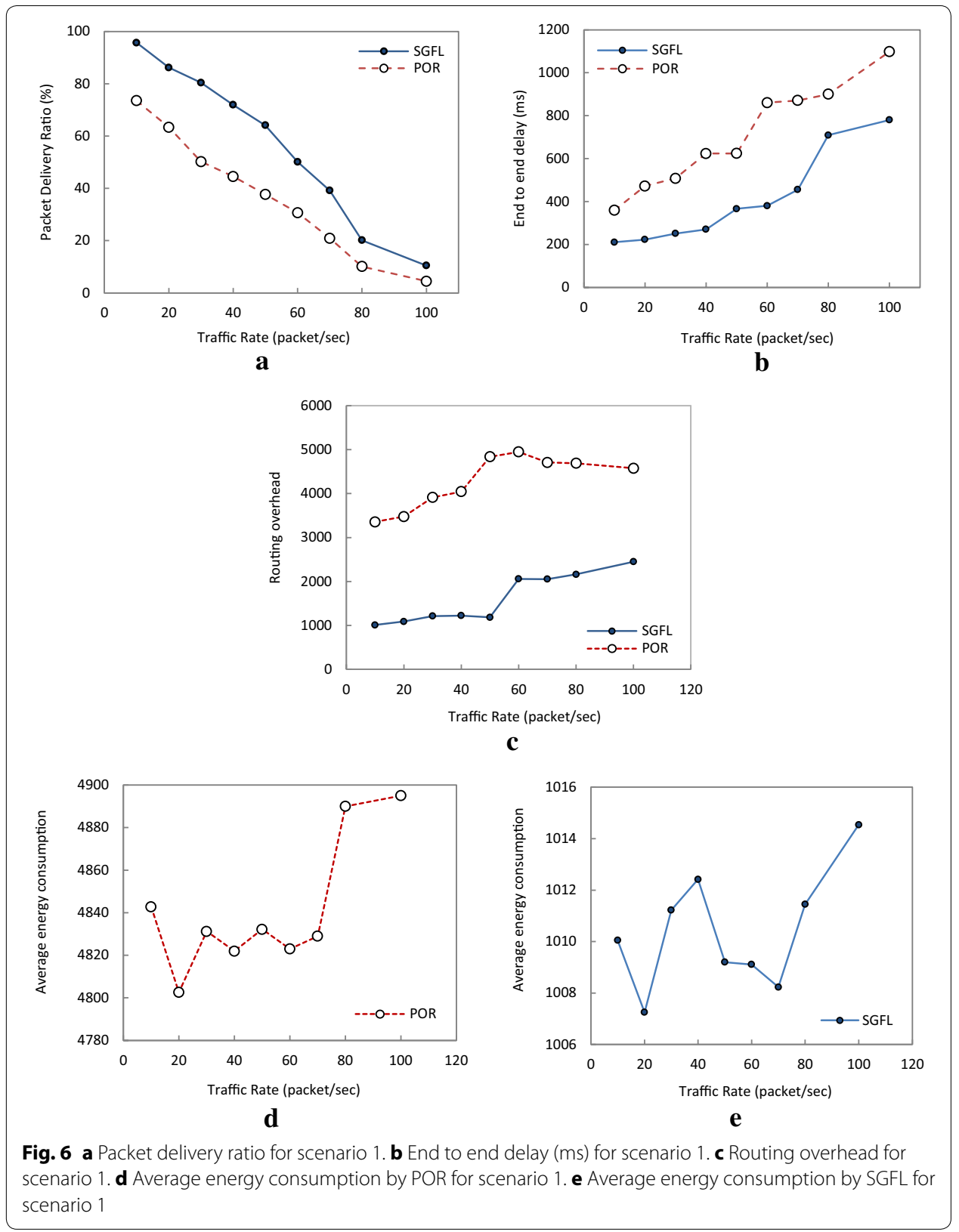

frequency increases, routing overhead is increased for both protocols. In case of SGFL routing overhead is lower than POR because our SGFL protocol allows only one backup node with each selected next hop that decrease the possibility of nodes out of range which reduces packet loss during the transmission. In Figs. 6(d, e) and 7d variation of network traffic rate with respect to average energy consumption is highlighted. As number of CBR packet frequency increases, energy consumption is increased slowly for both protocols. In group mobility, all nodes belonging to a particular group are often found closely connected to all other members of that group. This compels POR to choose more nodes in backup as compared to proposed mechanism employed with SGFL. 


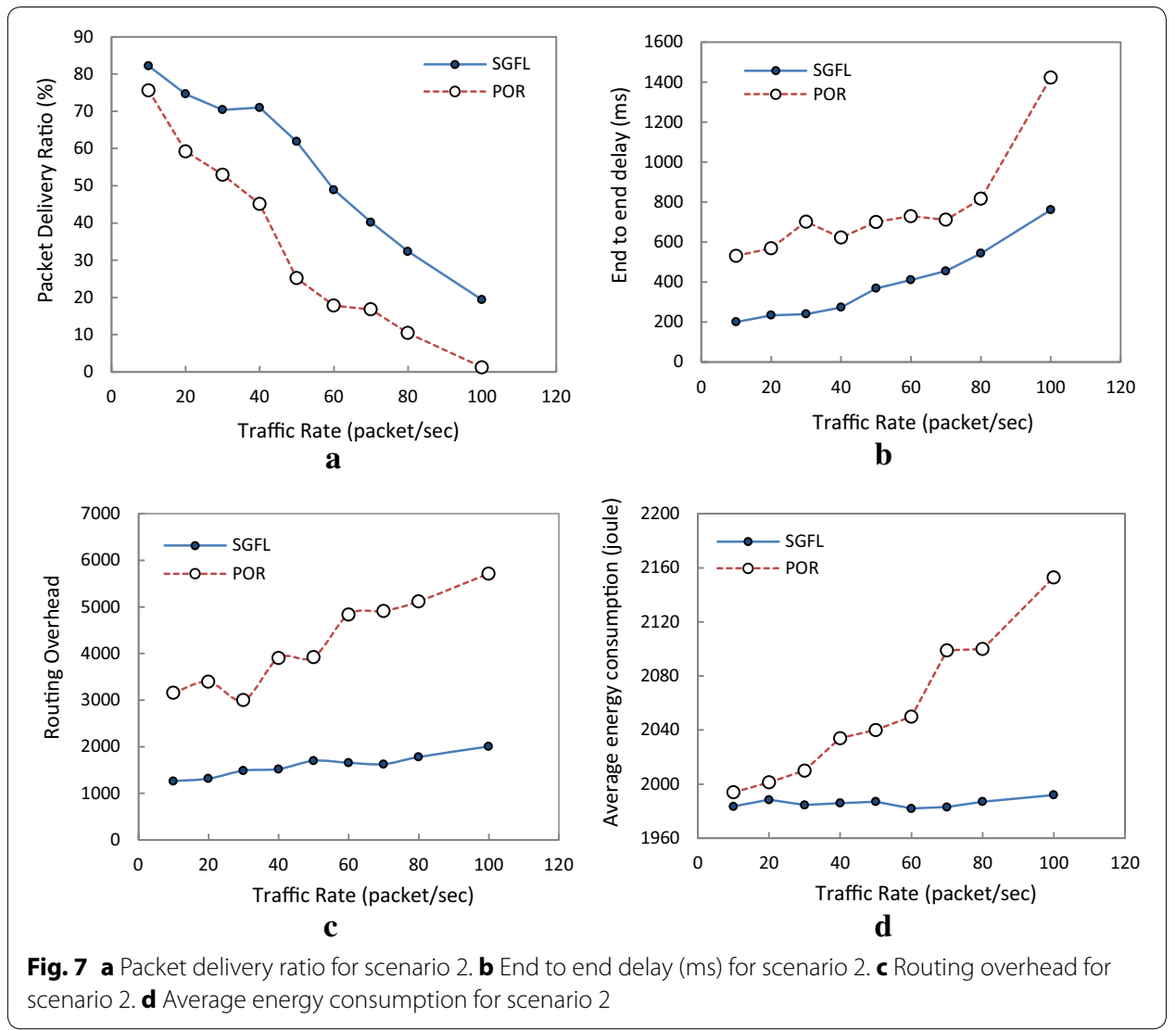

Impact of node speed on individual and group mobility scenarios

This section deals with the study of the impact of node speed on group mobility (Fig. 8) and random individual node mobility (Fig. 9) scenario, respectively. For this set of simulation, the source node transmits packets at a frequency of 50 packets/s. The variation in the network performance was obtained as node speed varied from 10 to $70 \mathrm{~m} / \mathrm{s}$ with pause time of $1 \mathrm{~s}$.

Figures $8 \mathrm{a}$ and $9 \mathrm{a}$ depicts the relationship of packet delivery ratio with respect to node speed for both the protocols. Decrease in the trend is observed because of the fact that link fails easily as node speed increases. However, the rate of decrease of packet delivery ratio for SGFL is obviously higher than that of POR. That is because POR is distance based routing protocol, link failure often occurs due to node mobility, and data packets are dropped upon experiencing a link failure. Figures $8 \mathrm{~b}$ and $9 \mathrm{~b}$ highlights the comparison of delays for SGFL and POR protocols. It is evident from the experimental outcome that, delays vary as node speed increases. That is because as node speed increases, topology changes faster, which more easily leads to link failure and triggers route rediscovery process, thereby raising an increase in transmission delay. However, the routing protocols indicate different performances. SGFL has smaller variation in end to end delay than POR, which indicates an improvement in transmission delay as node speed increases. Figures $8 \mathrm{c}$ and $9 \mathrm{c}$ shows the routing overhead with the increase of node mobility. Routing overhead is increased for both protocols as the node speed increases. However routing overhead of SGFL slower than POR. The reason behind this is the 


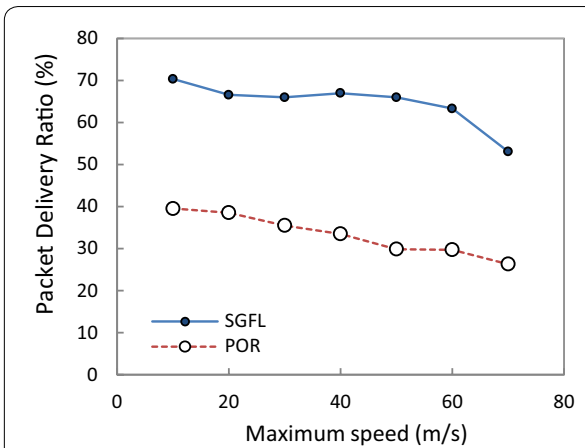

a

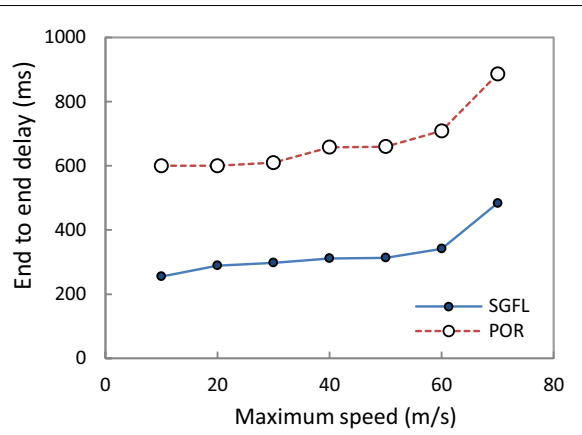

b

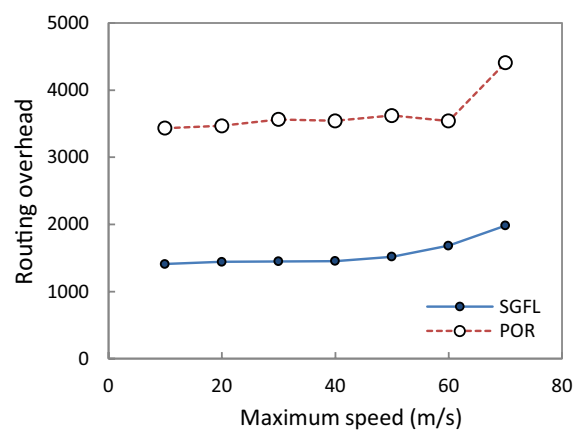

c
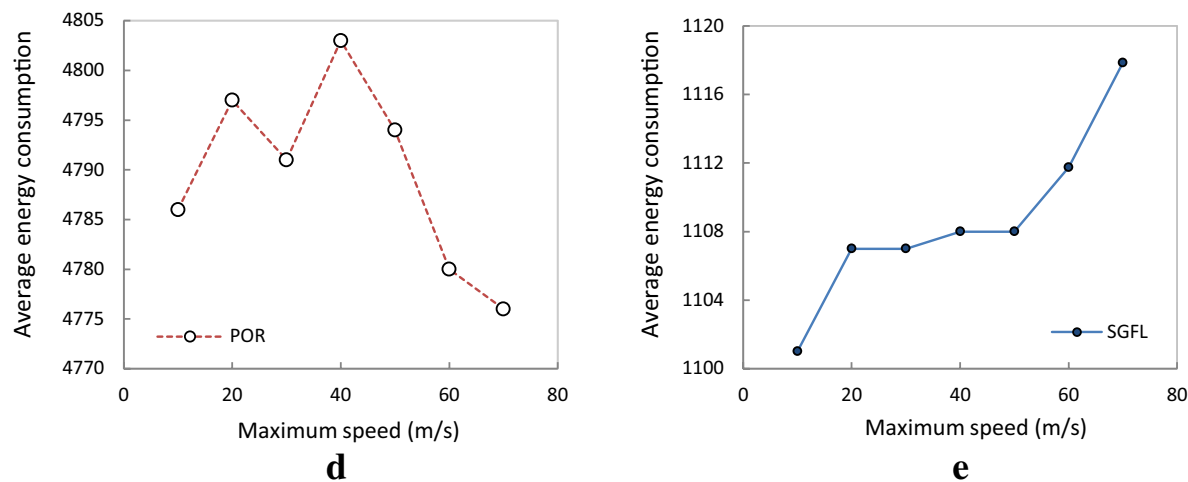

Fig. 8 a Packet delivery ratio for scenario 1. b End to end delay (ms) for scenario 1. c Routing overhead for scenario 1. d Average energy consumption by POR for scenario 1. e Average energy consumption by SGFL for scenario 1

novel methodology employed by proposed SGFL in selection of next hop forwarder and backup node. This imparts stability to inter-node communication by decreasing the possibility of packet loss during transmission. The results in Figs. 8(d, e) and 9d show that our protocol SGFL dominates over POR with respect to average energy consumption, even when the node speed increases.

\section{Comparative analysis over multiple routing protocols for random network scenario}

This section highlights a broader analysis of our proposed SGFL mechanism with existing list of counterpart protocols, including HARP [17], POR [18], and LBR [19] respectively. Figure 10 provides a collaborative study of the performance of proposed SGFL with above stated protocols in terms of average successful packet delivery ratio as rate of 


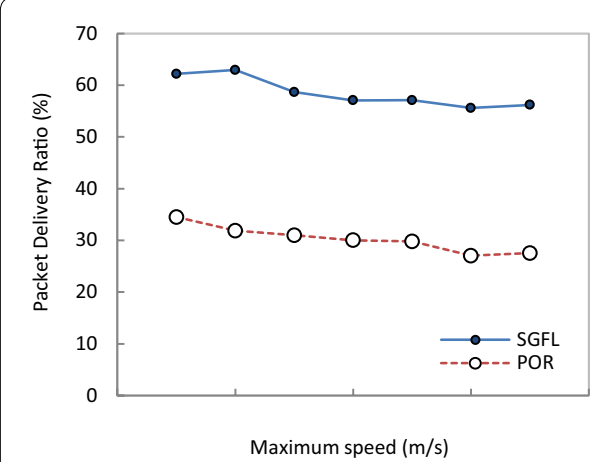

a

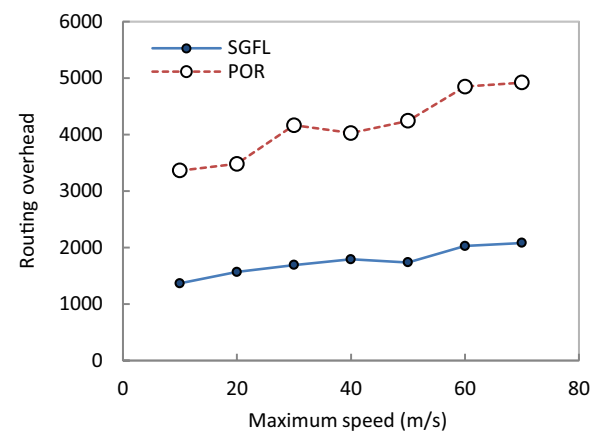

c

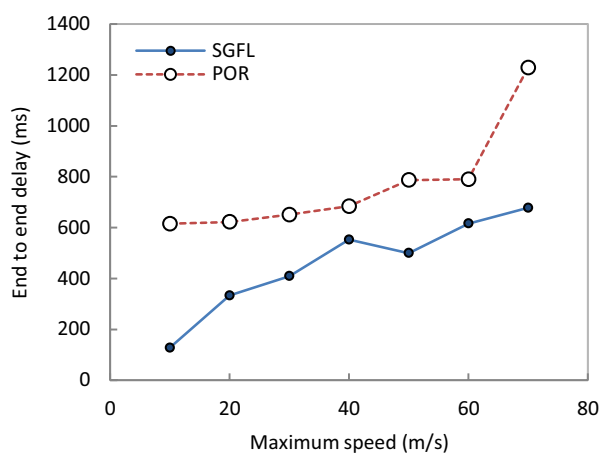

b

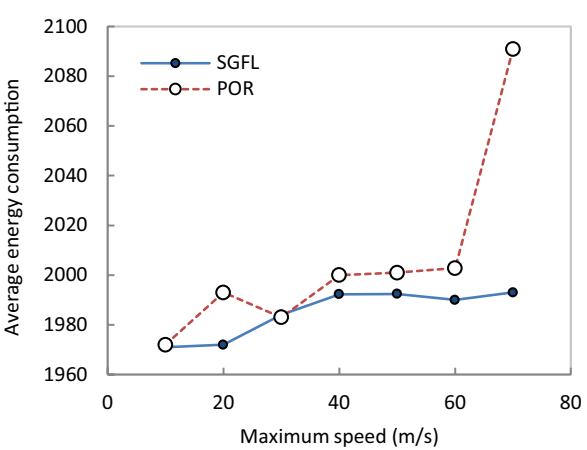

d

Fig. 9 a Packet delivery ratio for scenario 2. b End to end delay (ms) for scenario 2. c Routing overhead for scenario 2. d Average energy consumption for scenario 2

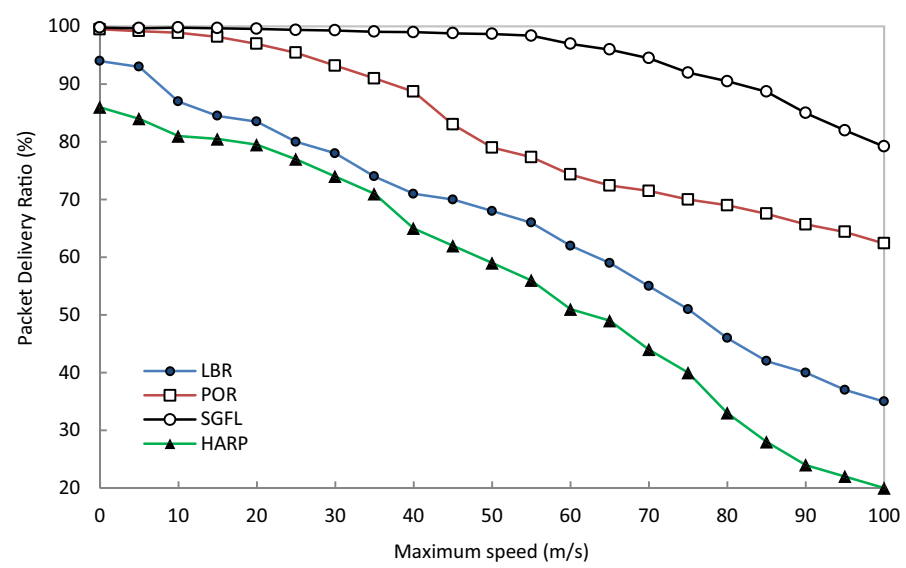

Fig. 10 Comparative study of packet delivery performance for scenario 3

mobility, i.e. speed of node movement, increases, in a scenario with randomly positioned nodes. This is different from above mentioned scenarios and hence is referred to as scenario 3. In this scenario nodes are randomly located and their relative speed may differ. Moreover, some of the nodes might have no mobility at all, thereby resulting in a hybrid scenario where half of the fraction of nodes may have speeds ranging around $100 \mathrm{~m} / \mathrm{s}$, 
while reaming nodes choose to remain at stationary position. As evident from Fig. 10, HARP employs node directivity as the sole measure to channel packets over reliable communication path with reduced routing overhead. However, heading-direction angles alone fails to appropriately predict the quality and longevity of communication link. Further, in LBR protocol two types of communication paths are built before transmission takes place. The first route acts as primary shortest path between the sender node and its corresponding destination, which is setup by flooding control messages. Additionally, backup paths are constructed with respect to each node in the primary route. With such exhaustive setting up of paths, the protocol claims considerable improvement of link lifetime and successful data delivery. However, extensive exchange of control messages owing to flooding during primary path construction, and existence of multiple backup paths; drastically degrades the service delivery performances with increase in node mobility.

POR is another existing counterpart that performs comparatively better than HARP and LBR in performance. This mechanism allows sender node to maintain a set of backup nodes, instead of building entire backup paths. Though, the routing overhead is comparatively reduced to some extent, but determining the optimal number of backup nodes plays an important trade-off between performance and routing (and buffer) overhead. Also, this approach does not takes into account the impact of network parameters in case of group mobility. Our proposed SGFL scheme addresses the above challenges and trade-offs while imparting performance to dynamic scenarios, involving individual and group mobility. SGFL is equipped with link stability metric that selects the next forwarder node having least distance and low mobility with respect to nodes lying in the progress area of source node. In addition, single backup node is associated with the forwarder to ensure continuation of network services even in case of link failure without inducing further delays. This perfectly balances the trade-off among performance in transmission and associated overheads in routing and resource utilization.

\section{Conclusion}

Research in this paper proposes a novel SGFL mechanism that performs geographic routing and link quality analysis with backup node selection. The usage of link lifetime prediction computed in terms of least distance and low mobility while choosing next hop forwarder; greatly contributed to increase communication reliability. Also, we adopt a backup node scheme to avoid dead node problem as well as link breakage and failure, thereby increasing lifetime of the communication route. The results obtained from performance analysis confirmed the dominance of our proposed SGFL over POR in both scenarios, involving group mobility and individual random mobility. Our research showed 30-40\% increase in the packet delivery ratio for congested networks and 10-20\% for high mobility networks. Moreover, the results indicated significant improvement in packet delivery ratio for different CBR intervals and node speed when compared to POR. Similarly, constructive outcomes were reflected from the simulation of the proposed methodology in terms of reduced routing overhead, end-to-end delay, and energy consumption. Furthermore, it can be concluded that our proposed SGFL protocol would deliver better performance when implemented on battlefield environment. As future enhancements, improved mechanisms embedded in battlefield heavy 
vehicles can be constructed for sensor nodes, which can support power generator modules to harvest energy from natural sources. Also, impact of different realistic mobility models can be taken into consideration for high and low traffic scenario. Moreover, establishing secured communication for achieving reliability in battlefield services, could be another promising extension.

\section{Authors' contributions}

ADI participated in the design of models and their conceptual working, eventually leading to developing algorithms. PJ designed scenarios, implemented algorithms and generated output, along with preparing initial drafts. ADI upgraded results with comparative study, laid foundation for interpretive conclusion from outcomes and outlined manuscript with critical revision. Both, $\mathrm{ADI}$ and PJ participated in debugging and code refinement process while implementation. Both authors read and approved the final manuscript.

\section{Authors' information}

Priyanka Jaiswal received her Master of Technology in Computer Science in 2014 from Jaypee Institute of Information Technology, Noida, India. Her major research interest lies in wireless network research including, energy-aware routing in mobile adhoc network, network protocol development, mobility modelling and management.

Dr. Adwitiya Sinha was awarded with philosophy of doctorate in computer science from Jawaharlal Nehru University (JNU), New Delhi, India in 2015. During her research in wireless sensor networks, she was honoured with Senior Research Fellowship (SRF) from Council of Scientific and Industrial Research (CSIR), New Delhi. She received Master of Technology in Computer Science in 2010 from the same university. She also completed Master and Bachelor programmes in Computer Applications in 2008 and 2006, respectively. Presently she is working as Assistant Professor (Senior Grade) in Jaypee Institute of Information Technology, Noida, India. She is actively involved in wireless network research, especially in areas including, energy-efficient wireless routing protocols, data aggregation, multi-layer fusion techniques, intelligent traffic pattern recognition and other new generation computing technologies with social network integration.

\section{Competing interests}

Both authors declare that they have no competing interests.

Received: 7 October 2014 Accepted: 18 September 2016

Published online: 10 December 2016

\section{References}

1. Mauve M, Widmer J, Hartenstein H (2001) A survey on position based routing in mobile adhoc networks. IEEE Network 15(6):30-39

2. Mohandas G, Silas S, Sam S (2013) Survey on routing protocols on mobile adhoc networks. In: Proceedings of IEEE international multi-conference on automation, computing, communication, control and compressed sensing (iMac4s), pp 514-17

3. Govindaswamy V, Blackstone WL, Balasekaran G (2011) Survey of recent position based routing mobile ad hoc network protocol. In: Proceedings of IEEE 13th international conference on computer modeling and simulation, pp 467-71

4. Garcia M, Ladiod H (2007) A performance comparison of position based routing approaches for mobile adhoc networks. In: Proceedings of 66th IEEE international conference on vehicular technology, pp 1-5

5. Lenders V, Wagner J, Heimlicher S, May M, Plattner B (2008) An empirical study of the impact of mobility on link failures in an 802.11 ad hoc network. IEEE Wirel Commun 15(6):16-21

6. Long J, Dong M, Ota K, Liu A (2014) Achieving source location privacy and network lifetime maximization through tree-based diversionary routing in wireless sensor networks. IEEE Access 2:633-651

7. Chen Q, Kanhere SS, Hassan M (2013) Adaptive position update for geographic routing in mobile adhoc networks. IEEE Trans Mob Comput 12(3):489-501

8. YU HY, LUYL (2011) Intelligent sensor network simulation for battlefield resources management. In: IEEE ninth international conference on dependable, autonomic and secure computing, pp 238-41

9. Li YX, Shi HS, Zhang SP (2010) An efficient multihop routing scheme in the large scale battlefield wireless sensor network. In: 3rd international conference on advanced computer theory \& engineering, pp 634-638

10. Alsharabi N, Fa LR, Zing F, Ghurab M (2008) Wireless sensor networks of battlefields hotspot: challenges and solutions. 6th IEEE international symposium on modeling and optimization in mobile, ad hoc, and wireless networks and workshops, pp 192-96

11. Walker III TO, Tummala M, McEachen J (2010) Energy efficiency of centralized and distributed computation in unattended multi-hop wireless sensor networks for battlefield monitoring. In: 43rd IEEE Hawaii international conference on system sciences, pp 1-9

12. Li YX, Shi HS, Zhang SP (2010) An optimized scheme for battlefield target tracking in wireless sensor network. In: 2nd IEEE international conference on industrial and information systems, pp 428-31

13. Jung S, Lee D, Yoon S (2008) A geographic routing protocol utilizing link lifetime and power control for mobile adhoc networks. In: Proceedings of 1 st ACM international workshop on foundations of wireless adhoc and sensor networking and computing, pp 25-32 
14. Rao SA, Pai MM, Boussedjra M, Mouzna J (2008) GPSR-L: greedy perimeter stateless routing with lifetime for VANETS In: proceedings of IEEE 8th international conference on ITS telecommunication, pp 299-304

15. Noureddine H, Ni Q, Min G, Al-Raweshidy H (2014) A new link lifetime estimation method for greedy and contention-based routing in mobile ad hoc networks. Telecomun Syst 55(3):421-433

16. Abdoos M, Faez K, Sabaei M (2009) Position based routing protocol with more reliability in mobile adhoc network. In: Proceedings of IEEE first Asian Himalayas international conference on internet, pp 1-4

17. Al-Akaidi M, Alchaita M (2007) Link stability and mobility in adhoc wireless networks. IET Commun 1(2):173-178

18. Yang S, Yeo CK, Lee BS (2012) Toward reliable data delivery for highly dynamic mobile adhoc networks. IEEE Trans Mob Comput 11(1):112-124

19. Yang W, Yang X, Yang S (2009) A stable backup routing protocol based on link lifetime in mobile adhoc networks. In: Proceedings of the IEEE 3rd international conference on mobile ubiquitous computing, systems, services and technologies, pp 202-07

20. Chen Y, Wang G, Peng S (2008) Link lifetime-based segment-by-segment routing protocol in MANETs. In: International IEEE symposium on parallel and distributed processing with applications, pp 387-92

21. Rao GM, Baig MN, Baba MF, Kumar KK (2011) Energy efficient reliable routing protocol for mobile adhoc networks. In: 3rd IEEE international conference on electronics computer technology, vol 5, pp 296-99

22. Bansal A, Singh M (2012) Enhancing MANET's performance: a cross-layer solution. In: 2nd IEEE international conference on parallel distributed and grid computing, pp 407-12

23. Fall F, Varadhan K (2009) The NS manual. VINT Project. http://www.isi.edu/nsnam/ns/doc/index.htm/

24. Issariyakul T, Hossain E (2009) Introduction to network simulator NS2. Springer, US. ISBN: 978-0-387-71759-3

25. Altman E, Jemenez T (2003) NS simulator for beginners. Morgan \& Claypool Publishers. ISBN: 9781608456925

\section{Submit your manuscript to a SpringerOpen ${ }^{\circ}$ journal and benefit from:}

- Convenient online submission

- Rigorous peer review

- Immediate publication on acceptance

Open access: articles freely available online

- High visibility within the field

- Retaining the copyright to your article

Submit your next manuscript at $\boldsymbol{\nabla}$ springeropen.com 